\title{
Intelligent Automatic Landing System Using Fuzzy Neural Networks and Genetic Algorithm*
}

\author{
Jih-Gau Juang Kuo-Chih Chin Jern-Zuin Chio \\ Department of Communications and Guidance Engineering \\ National Taiwan Ocean University, Keelung, Taiwan 20224 \\ jgjuang@mail.ntou.edu.tw
}

\begin{abstract}
In this paper, an intelligent automatic landing system using fuzzy neural networks and genetic algorithms is developed to improve the performance of conventional automatic landing systems. This study uses a functional fuzzy neural network as the controller. Control gains are selected by a combination method of a nonlinear control design and genetic algorithm. The simulation results are described for the automatic landing system of a commercial airplane. Tracking performance and robustness are demonstrated through software simulations. Simulation results show that the proposed scheme can successfully expand the safety envelope of an aircraft to include severe wind disturbance environments.
\end{abstract}

\section{Introduction}

From 1964 to 1982, 27 US aircraft accidents or incidents involving nearly 500 fatalities have been attributed to low-altitude wind shear [1]. According to NTSB (National Transportation Safety Board) statistics [2], between 1989 and 1999, there were approximately $22.6 \%$ aircraft accidents identified as wind shear. By causing a sudden divergence in wind direction or speed, wind shear creates a particular hazard for airplanes at takeoff and landing because the pilot is confronted with a rapid and unexpected shift from headwind to tailwind. An Aircraft flying through a wind shear first encounters a headwind that generates an extra lift. Shortly thereafter, the headwind is replaced by a tailwind that causes a sudden loss of lift. Hence, an inadvertent encounter with a low-altitude wind shear can be a serious problem even for a skilled pilot. In some cases, pilots will try to abort landing or escape the disturbance [3-4]. For those pilots that are unable to abort landing must face the disturbance and handle the aircrafts manually. It is therefore desirable to develop an intelligent Automatic Landing System (ALS) that expands the operational envelope to include more safe responses under severe wind conditions.

The earliest automatic pilots could do no more than maintain an aircraft in straight and level flight by

* This work was supported by the National Science Council, Taiwan, ROC, under Grant NSC91-2212-E-019-005. controlling pitch, yaw, and roll movements; and they are still used most often to relieve the pilot during routine cruising. Modern automatic pilots can, however, execute complex maneuvers or flight plans, bring aircraft into approach and landing paths. The first ALS was made in England in 1965. Since then, most aircraft have been installed with this system. The ALS relies on the Instrument Landing System (ILS) to guide the aircraft into the proper altitude, position, and approach angle during the landing phase. Conventional automatic landing systems can provide a smooth landing which is essential to the comfort of passengers. However, these systems work only within a specified operational safety envelope. When the conditions are beyond the envelope, such as turbulence or wind shear, they often cannot be used. Most conventional control laws generated by the ALS are based on the gain scheduling method [5]. Control parameters are preset for different flight conditions within a specified safety envelope which is relatively defined by Federal Aviation Administration (FAA) regulations. According to FAA regulations, environmental conditions considered in the determination of dispersion limits are: headwinds up to 25 knots; tailwinds up to 10 knots; crosswinds up to 15 knots; moderate turbulence, wind shear of 8 knots per 100 feet from 200 feet to touchdown [6]. If the flight conditions are beyond the preset envelope, the ALS is disabled and the pilot takes over. An inexperienced pilot may not be able to guide the aircraft to a safe landing.

Recently, many researchers have applied intelligent concepts such as neural networks, fuzzy systems, and genetic algorithms [7-12] to flight control to adapt to different environments. For ALS, most of the improvements have been on the guidance instruments, such as the GNSS Integrity Beacons, Global Positioning System, Microwave Landing System and Autoland Position Sensor [13-16]. By using improvement calculation methods and high accuracy instruments, these systems provide more accurate flight data to the ALS to make the landing more smooth. However, these researches did not include weather factors such as wind disturbances. There have been some researches on the problem of intelligent landing control [17-21] but most of them do not consider wind disturbances. Here, we present a learning scheme, which uses a fuzzy controller with genetic algorithm, to guide the aircraft to a safe landing and make the controller more 
robust and adaptive to the ever-changing environment.

\section{Automatic Landing Control}

A typical automatic landing system [22] uses a radio beam directed upward from the ground at $2.5^{0}$ to $3.5^{\circ}$, with equipment onboard the aircraft to measure the angular deviation from the beam and compute the perpendicular displacement of the aircraft from glide path. Additional equipment is used to provide azimuth information, so that the aircraft can be lined up with the runway. When the aircraft approaches the outer airport marker, which is about 4 nautical miles from the runway, the glide path signal is intercepted. The glide path will usually be intercepted at about 1200 to $1500 \mathrm{ft}$ altitude, with an airspeed of 145 to 150 knots (245 to $253 \mathrm{ft} / \mathrm{s}$ ), and then the automatic control system will be engaged. As the aircraft descends along the glide path, its pitch, attitude and speed must be controlled. The aircraft maintains a constant speed along the flight path. The descent rate is about $10 \mathrm{ft} / \mathrm{sec}$ and the pitch angle is between -5 to 5 degrees. As the aircraft descends 20 to 70 feet above the ground, the glide path control system is disengaged and a flare maneuver is executed. The vertical descent rate is decreased to $2 \mathrm{ft} / \mathrm{sec}$ so that the landing gear may be able to dissipate the energy of the impact at landing. The pitch angle of the airplane is then adjusted, between 0 to 5 degrees for most aircraft, which allows a soft touchdown on the runway surface.

A simplified model of a commercial aircraft that moves only in the longitudinal and vertical plane was used in the simulations for implementation ease [15]. The motion equations for the aircraft are given as follows

$$
\begin{aligned}
\dot{u}= & X_{u}\left(u-u_{g}\right)+X_{w}\left(w-w_{g}\right)+X_{q} q-g\left(\frac{\pi}{180}\right) \cos \left(\gamma_{0}\right) \\
& +X_{E} \delta_{E}+X_{T} \boldsymbol{\delta}_{T} \\
\dot{w}= & Z_{u}\left(u-u_{g}\right)+Z_{w}\left(w-w_{g}\right)+\left(Z_{q}-\frac{\pi}{180} U_{0}\right) q \\
& -g\left(\frac{\pi}{180}\right) \sin \left(\gamma_{0}\right) \boldsymbol{\theta}+Z_{E} \boldsymbol{\delta}_{E}+Z_{T} \boldsymbol{\delta}_{T} \\
\dot{q}= & M_{u}\left(u-u_{g}\right)+M_{w}\left(w-w_{g}\right)+M_{q} q+M_{E} \delta_{E}+M_{T} \delta_{T} \\
\dot{\boldsymbol{\theta}}= & q \\
\dot{h}= & -w+\frac{\pi}{180} U_{0} \boldsymbol{\theta}
\end{aligned}
$$

where $u$ is the aircraft longitudinal velocity $(\mathrm{ft} / \mathrm{sec}), w$ is the aircraft vertical velocity ( $\mathrm{ft} / \mathrm{sec}), q$ is the pitch rate ( $\mathrm{deg} / \mathrm{sec}), \boldsymbol{\theta}$ is the pitch angle (deg), $h$ is the aircraft altitude (ft), $\boldsymbol{\delta}_{E}$ is the incremental elevator angle (deg), $\boldsymbol{\delta}_{T}$ is the throttle setting $(\mathrm{ft} / \mathrm{sec}), \gamma_{0}$ is the flight path angle $(-3 \mathrm{deg})$, and $g$ is the gravity $\left(32.2 \mathrm{ft} / \mathrm{sec}^{2}\right)$. The parameters $X_{i}, Z_{i}$, and $M_{i}$ are the stability and control derivatives.

To make the ALS more intelligent, reliable wind profiles are necessary. Two wind disturbance models are most common in aircraft flight paths. They are turbulence and wind shear. In this paper, we put focus on wind shear since it is the most difficult condition to deal with during aircraft landing. A discretized approximation model for wind shear was used. The longitudinal wind velocity is given by

$W_{x}=\left\{\begin{array}{cc}-k & x \leq a \\ -k+2 k \frac{x-a}{b-a} & a<x<b \\ k & x>b\end{array}\right.$

and the vertical wind velocity is given by

$W_{h}=\left\{\begin{array}{cc}-k\left[\frac{h}{h_{0}}\right]\left[\frac{x-a}{c-a}\right] & a \leq x \leq c \\ -k\left[\frac{h}{h_{0}}\right]\left[\frac{b-x}{b-c}\right] & c \leq x \leq b \\ 0 & x \geq b\end{array}\right.$

where $a, b, c=\frac{a+b}{2}$ are various horizontal distance measured from the initial position, $h_{0}$ is a reference altitude, and $k$ is a measure of the wind shear intensity. Figure 1 shows two wind shear profiles with $k=40$ (dash line) and $k$ $=50$ (solid line).
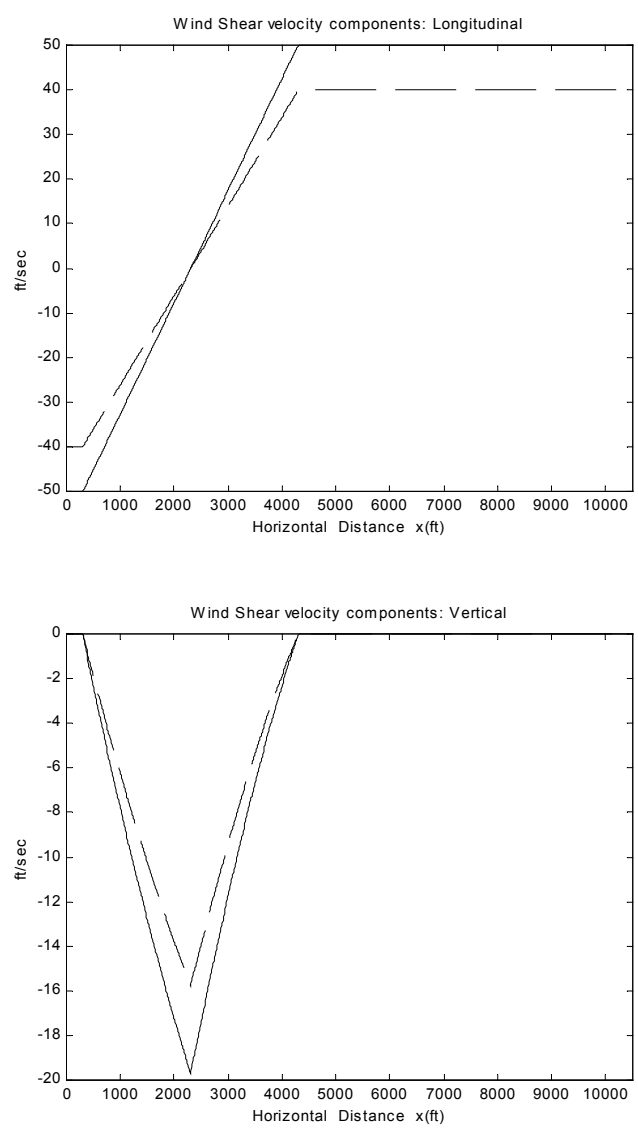

Figure 1. Wind shear profiles. 


\section{Fuzzy Controller}

Since the aircraft maintains a constant speed along the flight path, we assumed that the change in throttle command is zero. The aircraft is thus controlled solely by the pitch command. Detailed descriptions can be found in [15]. A complete landing phase is divided into several stages (intervals). Each stage uses the same fuzzy controller. Wind disturbances are added to each stage in the simulation. The inputs, $x_{i}$, for the fuzzy controller are: altitude, altitude command, altitude rate, and altitude rate command. The output of the controller is the pitch command. Figure 2 shows the structure of the fuzzy neural network.

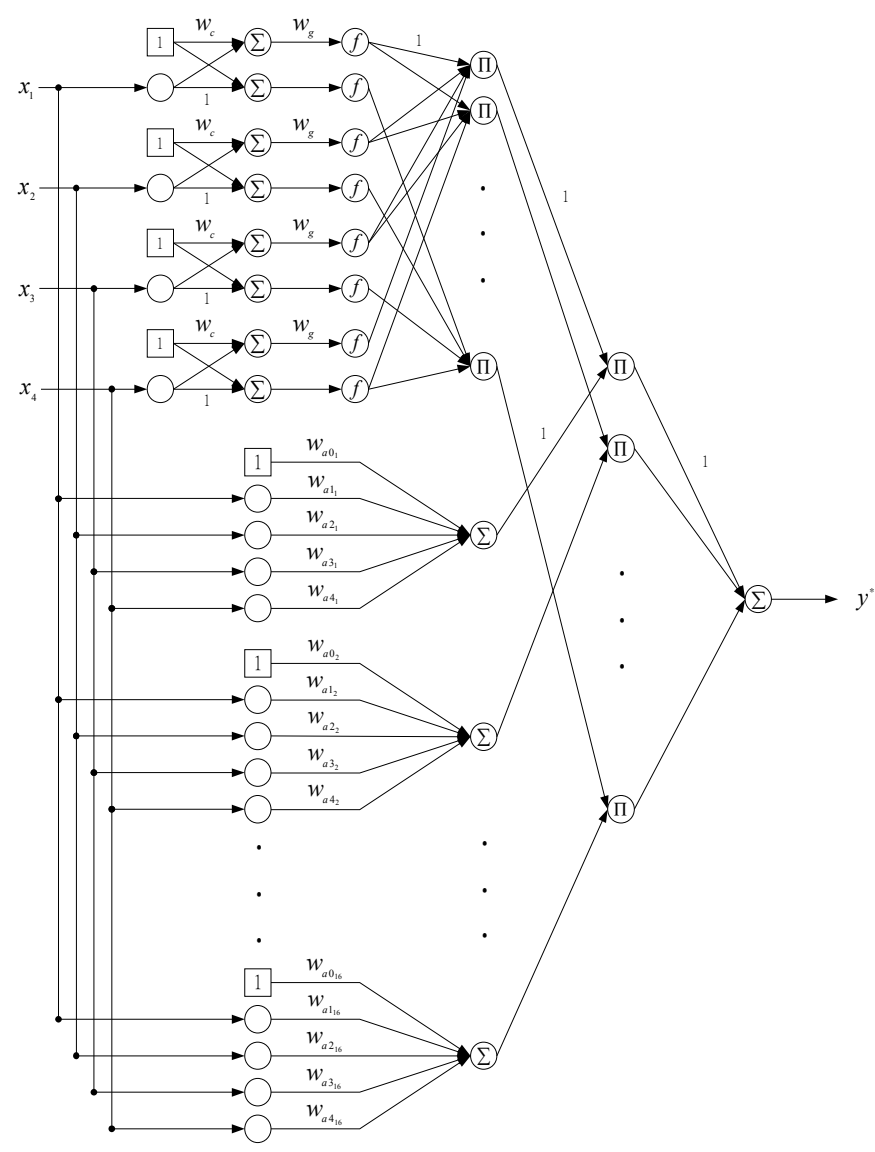

Figure 2. Fuzzy network with functional rule.

Compare to the linguistic rule, functional rule has more variable in the consequence. The order of the dimension is proportional to the number of inputs. The network realizes the following inference method:

$$
\begin{aligned}
& R^{i}: \text { IF } x_{1} \text { is } A_{1_{i 1}} \text { and } x_{2} \text { is } A_{2_{i 2}} \text { and... and } x_{4} \text { is } A_{4_{i 4}} \\
& \text { then } y \text { is } \varphi_{i}\left(x_{1}, x_{2}, x_{3}, x_{4}\right)
\end{aligned}
$$

where $i 1, i 2, i 3$, and $i 4$ is from 1 to 2 ; $i$ is from 1 to 16 . The function in consequence of the fuzzy rule can be any polynomial. In here we use first order linear equation:

$\varphi_{i}\left(x_{1}, x_{2}, x_{3}, x_{4}\right)=a_{1} x_{1}+a_{2} x_{2}+a_{3} x_{3}+a_{4} x_{4}$

where $a_{i}$ is a scalar and adjusted during network training.
Learning process of aircraft landing is shown in Figure 3. $\mathrm{FC}$ is the fuzzy controller. $\mathrm{CL}$ is the control law that used in [15]. AM is the aircraft model. $\boldsymbol{C}_{i}$ is the pitch command. The connection weights are modified to identify fuzzy rules and tune the membership functions in the premises using the following backpropagation algorithm:

1. at sixth layer

$$
\delta^{6}=(d-y) f^{\prime}\left(I^{6}\right)=d(n)-y(n)
$$

2. at fifth layer

$$
\delta_{i}^{5}=f^{\prime}\left(I_{i}^{5}\right) \sum_{k} \delta_{k}^{6} \cdot 1=\delta_{k}{ }^{6}, i=1,2 \ldots 16, k=1
$$

3. at fourth layer

$$
\begin{aligned}
& \delta_{i}^{4}=f^{\prime}\left(I_{i}^{4}\right) \sum_{k}\left(\delta_{k}^{5} \cdot \prod_{j \neq i} O_{j}^{4}\right)=\frac{1}{\sum \mu_{k}}\left(\delta_{k}^{5} \cdot \prod_{j \neq i} O_{j}^{4}\right) \\
& \delta_{i}^{4}=f^{\prime}\left(I_{i}^{4}\right) \sum_{k}\left(\delta_{k}^{5} \cdot \prod_{j \neq i} O_{j}^{4}\right)=\delta_{k}^{5} \cdot \prod_{j \neq i} O_{j}^{4} \\
& i=1,2 . .16, j=1,2 \ldots 4 \\
& w_{a_{j i}}(m+1)=w_{a_{j i}}(m)+\eta \delta^{4} x_{i} \\
& w_{a_{0 i}}(m+1)=w_{a_{0 i}}(m)+\eta \delta^{4} 1,
\end{aligned}
$$

4. at third layer

$$
\begin{aligned}
& \delta_{i}^{3}=f^{\prime}\left(I_{i}^{3}\right) \sum_{k}\left(\delta_{k}^{4} \cdot \prod_{j \neq i} O_{j}^{3}\right)=O_{3} \cdot\left(1-O_{3}\right) \cdot \sum_{k}\left[\delta_{k}^{4} \cdot \prod_{j \neq i} O_{j}^{3}\right] \\
& \quad k=1,2 \ldots . .8 \\
& w_{g}(m+1)=w_{g}(m)+\eta \delta^{3} O^{2} \\
& \text { 5. at second layer } \\
& \delta_{i}^{2}=f^{\prime}\left(I_{i}^{2}\right) \sum_{k} \delta_{k}^{3} w_{k i}^{3}=\delta_{i}^{3} \cdot w_{g}, \quad i=1,2 \ldots .8 \\
& w_{c}(m+1)=w_{c}(m)+\eta \delta^{2} O^{1}
\end{aligned}
$$

In the simulations, initial flight conditions are: $h(0)=500 \mathrm{ft}$, $\dot{x}(0)=235 \mathrm{ft} / \mathrm{sec}, x(0)=9240 \mathrm{ft}$, and $\gamma_{0}=-3$ degrees. With the wind shear intensity at 59 , the horizontal position at touchdown is $269 \mathrm{ft}$, horizontal velocity is $234.7 \mathrm{ft} / \mathrm{sec}$, vertical speed is $-2.4 \mathrm{ft} / \mathrm{sec}$, and pitch angle is -7 degrees, as shown in Figure 4 and 5. Table I shows the results from using different wind shear intensities with different control gains of the pitch autopilot.

\section{Fuzzy Control with GA}

In this section genetic algorithm is utilized to the selection of optimal control gains, which are used to make the controller adaptive to different flight conditions. Robustness is obtained by choosing optimal control gains that allows wide range of disturbances to the controller. Since GAs have the potential for global optimization, they are suitable for determination of the control parameters which give aircraft better adaptivity in severe environment. GAs are search and optimization algorithms based on the principle of natural evolution and population genetics. The basic principles of GAs were first proposed by Holland 


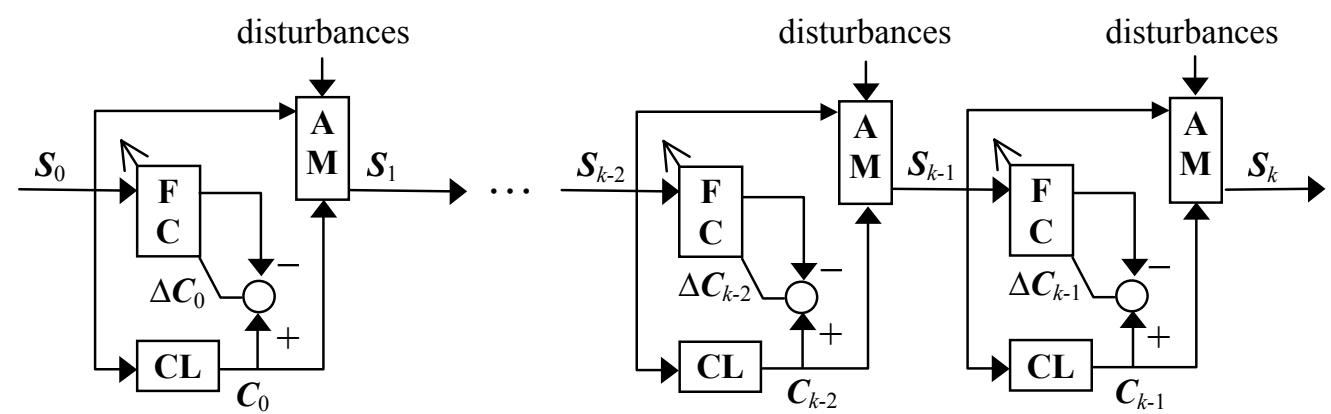

Figure 3. Learning process.

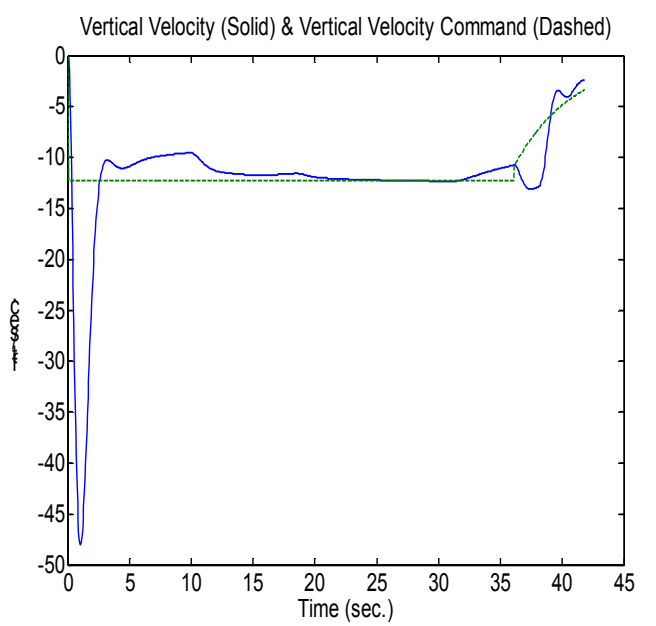

Figure 4. Aircraft vertical velocity and command.

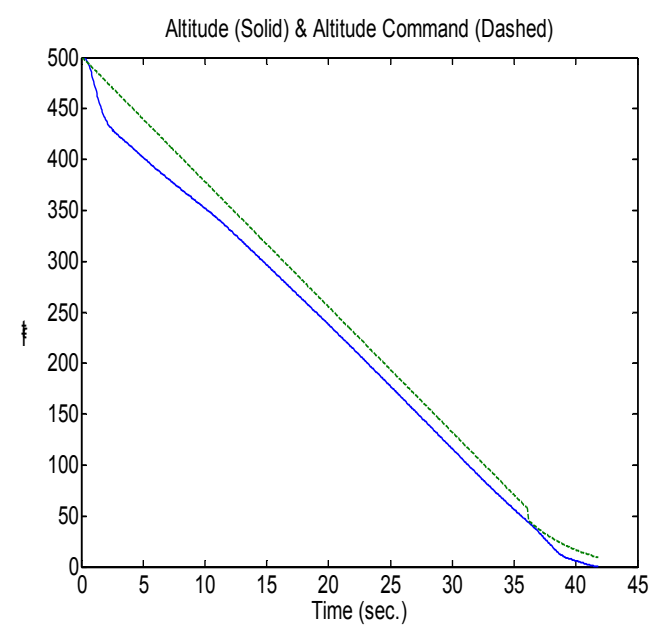

Figure 5. .Aircraft altitude and command.

Table I. The results from using different wind shear intensities.

\begin{tabular}{|l|c|c|c|c|c|}
\hline Wind shear intensity & 57 & 58 & 59 & 60 & 61 \\
\hline Landing point (ft) & -59 & 81 & 269 & 468 & 726 \\
\hline Aircraft vertical speed (ft/sec) & -3.3 & -2.6 & -2.4 & -2.5 & -2.3 \\
\hline Pitch angle (degree) & -9.9 & -9.9 & -7.1 & -10.0 & -6.9 \\
\hline
\end{tabular}

[23]. GA presumes that the potential solution of any problem is an individual and can be represented by a set of parameters. These parameters are regarded as the genes of a chromosome and can be structured by a string of values in binary form. A positive value, generally known as a fitness value, is used to reflect the degree of "goodness" of the chromosome for the problem which would be highly related with its objective value [24].

Throughout a genetic evolution, the fitter chromosome has a tendency to yield good quality offspring which means a better solution to any problem. In a practical GA application, a population pool of chromosomes has to be installed and these can be randomly set initially. In each cycle of genetic operation, termed as an evolving process a subsequent generation is created from the chromosomes in the current population. This can only succeed if a group of these chromosomes, generally called "parents" or a collection term "mating pool" is selected via a specific selection routine. The genes of the parents are mixed and recombined for the production of offspring in the next generation. It is expected that from this process of evolution (manipulation of genes), the "better" chromosome will create a larger number of offspring, and thus has a higher chance of surviving in the subsequent generation, emulating the survival-of-the-fittest mechanism in nature. A scheme called Roulette Wheel Selection [25] is one of the most common selection techniques and is used in this paper.

The cycle of evolution is repeated until a desired termination criterion is reached. This criterion can also be 
set by the number of evolution cycles (computational runs), or the amount of variation of individuals between different generations, or a pre-defined value of fitness. In order to facilitate the GA evolution cycle, two fundamental operators: Crossover and Mutation are required, although the selection routine can be termed as the other operator. An one-point Crossover mechanism is used in this paper. A crossover point is randomly set. The portions of the two chromosomes beyond this cut-off point to the right are to be exchanged to form the offspring. An operation rate $\left(p_{c}\right)$ with a typical value between 0.6 and 1.0 is normally used as the probability of crossover. However, for mutation, this applied to each offspring individually after the crossover exercise. It alters each bit randomly with a small probability $\left(p_{m}\right)$ with a typical value of less than 0.1 . The choice of mutation rate $p_{m}$ and crossover rate $p_{c}$ as the control parameters can be a complex nonlinear optimization problem to solve. Furthermore, their settings are critically dependent upon the nature of the objective function.

In here, population size is 20 , crossover rate is 0.8 , mutation rate is 0.01 . The fitness function is defined as

$$
f=\frac{w}{0.1+\left\|\overline{S_{d}}-\overline{S_{k}}\right\|}+s w,
$$

where $\overline{S_{d}}$ is the normalized desired state at touch down point, $\overline{S_{k}}$ is the normalized real state at touch down, $w$ is a weighting factor, and $s w$ is the strength of the allowable wind disturbance. The string of each chromosome has 32 bits which consists 8 bits of $k_{\theta}$ and $k_{q}$ in glide path and flare path. Initial conditions and searching rages of these four parameters are pre-selected by a nonlinear control design algorithm [26], which was developed from the Quasi-Newton method and Sequential Quadratic Programming method. With the wind shear intensity at 59, the horizontal position at touchdown is $269 \mathrm{ft}$, horizontal velocity is $235 \mathrm{ft} / \mathrm{sec}$, vertical speed is $-2.3 \mathrm{ft} / \mathrm{sec}$, and pitch

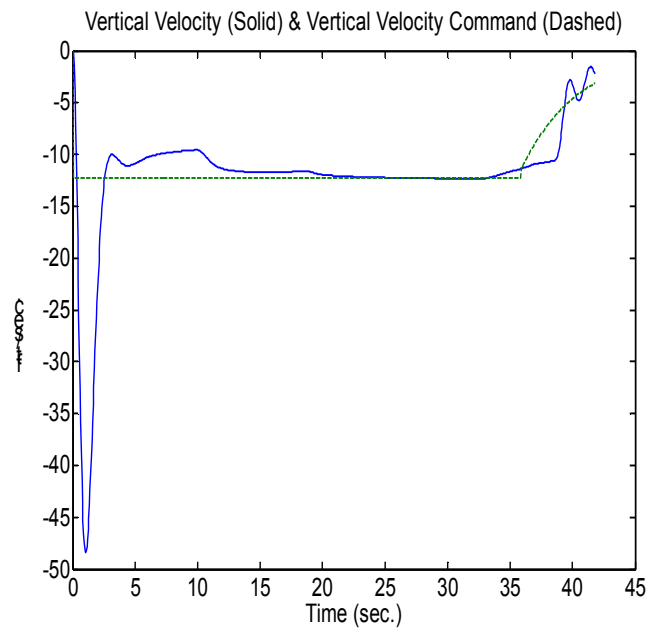

Figure 6. Aircraft vertical velocity and command.. angle is -9.9 degrees, as shown in Figure 6 to 7. Table II shows the results from using different wind shear intensities. The controller can successfully guide the aircraft flying through wind shear intensities of 49 to 60 .

\section{Conclusions}

The purpose of this study was to investigate the use of fuzzy neural networks and genetic algorithm in automatic landing systems and to make these systems more intelligent. Current flight control law was adopted in the intelligent controller design. Tracking performance and adaptive capability were demonstrated through software simulations. For the safe landing of an aircraft with a conventional controller, the wind shear intensity limit is 30 . In this study, the functional fuzzy controller with GA can overcome wind shear intensity to 60 . From these simulations, the proposed fuzzy controllers can successfully expand the controllable environment in severe wind disturbances.

\section{References}

[1] T.T. Fujita, "DFW Microburst," Satellite and Mesometeorology Research Project, 1986.

[2] NTSB Weather Related Accidents, fttp://www.asy.faa. gov/safety_analysis/weather_study/totals.

[3] A. Dogan and P.T. Kabamba, "Escaping a Microburst with Turbulence," Proc. American Control Conference, pp. 1349-1353, 2000.

[4] C.Y. Chen, "Optimal Abort Landing Trajectories for a High-Angle-Of-Attack Windshear Encounter," The Chinese Journal of Mechanics, vol. 11, no. 1, pp. 75-81, 1995.

[5] H. Buschek and A.J. Calise, "Uncertainty Modeling and Fixed-Order Controller Design for a Hypersonic Vehicle Model," Journal of Guidance, Control, and Dynamics, vol. 20, no. 1, pp. 42-48, 1997.

[6] Federal Aviation Administration, "Automatic Landing Systems,” AC 20-57A, Jan. 1971.

[7] A.J. Calise and R.T. Rysdyk, "Nonlinear Adaptive

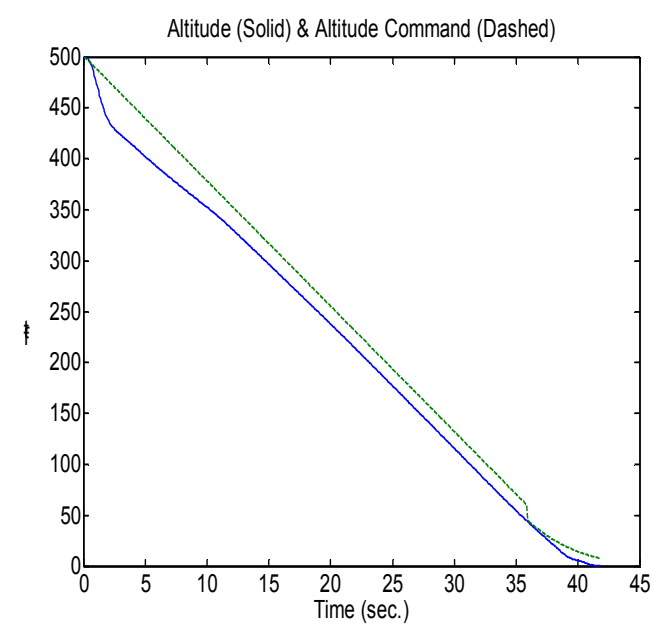

Figure 7. Aircraft altitude and command 
Table II. The results from using fuzzy controller with GA.

\begin{tabular}{|l|c|c|c|c|c|}
\hline Wind shear intensity & 49 & 50 & 55 & 59 & 60 \\
\hline Landing point (ft) & -305 & -270 & -94 & 269 & 433 \\
\hline Aircraft vertical speed (ft/sec) & -1.7 & -1.6 & -2.0 & -2.3 & -3.0 \\
\hline Pitch angle (degree) & -8.7 & -8.8 & -9.4 & -9.9 & -10 \\
\hline
\end{tabular}

Flight Control Using Neural Networks," IEEE Control Systems Magazine, vol. 18, no. 6, pp. 14-25, 1998.

[8] P.J. Werbos, "Neural Networks and Flight Control: Overview of Capabilities and Emerging Applications," Proc. Of the AIAA Guidance, Navigation, and Control Conf., pp. 912-919, 1995.

[9] M.R. Napolitano and M. Kincheloe, "On-Line Learning Neural-Network Controller for Autopilot Systems," Journal of Guidance, Control, and Dynamics, vol. 18, no. 5, pp. 1008-1015, 1995.

[10] B.C. Chen, "The Application of Neural Network on Longitudinal Flight Control System Design," M.S. thesis, National Chung Shing University, Taiwan, 1994.

[11] C.D. Shei, "Gain Scheduling of Flight Control System with Fuzzy Logic Model," M.S. thesis, National Chiao Tung University, Taiwan, 1996.

[12] C.L. Lin, "On the Design of an Adaptive Fuzzy Gain-Scheduled Autopilot," Proc. American Control Conference, pp. 1567-1572, 2002.

[13] C.E. Cohen, et al., "Automatic Landing of a 737 Using GNSS Integrity Beacons," Proc. ISPA, 1995.

[14] DDC-I, "Advanced Auto Landing System from Swiss Federal Aircraft Factory," Real-Time Journal, Sprint, 1995.

[15] S. Asai, et al., "Development of Flight Control System for Automatic Landing Flight Experiment," Mitsubishi Heavy Industries Technical Review, vol. 34, no. 3, 1997.

[16] D.N. Kaufmann and B.D. McNally, "Flight Test Evaluation of the Stanford University and United Airlines Differential GPS Category III Automatic Landing System," NASA Technical Memorandum 110354, June 1995.
[17] G. Saini and S.N. Balakrishnan, "Adaptive Critic Based Neurocontroller for Autolanding of Aircrafts with Varying Glideslopes," Proc. International Conference on Neural Networks, vol. 4, pp. 2288-2293, 1997.

[18] C.C. Jorgensen and C. Schley, "A Neural Network Baseline Problem for Control of Aircraft Flare and Touchdown," Neural Networks for Control, pp. 403-425, 1991.

[19] Y. Iiguni, H. Akiyoshi, and N. Adachi, "An Intelligent Landing System Based on Human Skill Model," IEEE Transactions on Aerospace and Electronic Systems, vol. 34, no. 3, pp. 877-882, 1998.

[20] Y. Li, N. Sundararajan, and P. Saratchandran, "Neural-Flight Controller for Aircraft Using Minimal RAN," Neural Computing and Applications, vol. 10, no. 2, pp. 172-183, 2000.

[21] H. Izadi, M. Pakmehr, and N. Sadati, "Optimal Neuro-Controller in Longitudinal Autolanding of a Commercial Jet Transport," Proc. IEEE International Conference on Control Applications, CD-000202, 2003.

[22] B.L. Stevens and F.L. Lewis, Aircraft Control and Simulation, New York: Wiley, 1992.

[23] J. H. Holland, Adaption in Natural and Artificial System, University of Michigan Press, 1975.

[24] K.F. Man, K.S. Tang, and S. Kwong, Genetic Algorithms, Springer, 1999.

[25] C.T. Lin and C.S. G. Lee, Neural Fuzzy Systems, Prentice Hall, 1996.

[26] J.G. Juang and M.D. Hwang, "Applications of Genetic Algorithm and PID control to a Nonlinear TRMS," Proc. Conference on Artificial Intelligence and Applications, pp. 734-739, 2002. (in Chinese) 